\title{
Medien und Medizin
}

\section{Erhard Taverna}

Nach einer zweijährigen Pause wurde Ende März 2007 im Kantonsspital Luzern das bekannte interdisziplinäre Forum mit dem Symposium «Medien und Medizin» unter Leitung des Chefarztes Prof. Dr. med. Peter Stulz wiederaufgenommen. Das hochgesteckte Ziel einer «Horizonterweiterung in der Medizin XXX» dürfte auch dieses Mal im stets bis zum letzten Platz besetzten Hörsaal erreicht worden sein. Kommunikations- und Medienwissenschaftler, Soziologen, Philosophen. Medienprofis und Ärzte vermittelten eine Einführung in das komplexe Thema massenmedialer Wechselwirkungen zwischen Medizin, Patient und Öffentlichkeit. Das berühmte Diktum von Marshall McLuhan (1911-1980) «The medium is the message», stand wie ein unsichtbarer Leitspruch über allen Erklärungsversuchen, Interpretationen und Analysen.

Gaetano Romano, Professor am Soziologischen Seminar der Universität Luzern, eröffnete den Zyklus mit seinen Überlegungen zur Wahrheit der Massenmedien. Am Beispiel des Buchdrucks seit Gutenberg lässt sich der soziologische Begriff der «funktionalen Differenzierung» als eine historische Folge fundamentaler Umstrukturierungen von Wissenssystemen erklären. Unkontrollierbare Verbreitung von Wissen, Autonomie des Lesers und nach langer Verzögerung auch der Leserin, Vernetzung der Interessenten, spezialisierte Publikationen, Expertenkultur und Akademisierung definieren einen Vorgang, der zahlreiche Gebiete wie Wissenschaft, Recht, Medizin und Politik erfasst. Mit jeder neuen Technologie wird ein Wissenssystem weiter ausdifferenziert und demokratisiert. Der Teilnehmerkreis erweitert sich, neue Berufe entstehen, und alte verlieren ihr Monopol, wohl nirgends ausgeprägter als im Gesundheitswesen mit seiner enormen Erweiterung seit den 1960er Jahren. Auf dem für alle offenen Marktplatz medialer Angebote ist ein Kampf um Aufmerksamkeit entbrannt. Besonders gut verkaufen lassen sich neue und kontroverse Themen. Gesucht sind Normverletzungen und die Abweichung von gesellschaftlichen Erwartungen. Komplexes wird auf Personen reduziert, unablässig hergestellte Betroffenheit sorgt für Beachtung und Absatz. Die Folge dieser Verkaufsstrategie ist eine endemische Beunruhigung durch Dramatisierung und Skandalisierung. Diese Klaviatur der öffent- lichen Erregung beeinflusst unsere Wahrnehmung von Risiken, was bis in die Gesetzgebung hineinwirkt. Dafür aktuelle Beispiele sind die Forderungen nach verschärften Massnahmen für Medikamentenversuche, Sterbehilfe, Sexualstraftäter oder Nahrungsmittelzusätze. Kurz für alles, was in den Tagesnachrichten als Gefahrenthema dominiert.

Roger de Weck lieferte in einem brillanten Referat das publizistische Fleisch am soziologischen Knochen. Er plädierte für die Rückkehr eines Journalismus, dem Zeit und Mittel für Recherchen zur Verfügung stehen, und geisselte mit Witz und Ironie den degradierten «content provider», der mit Banalitäten in Echtzeit der Pseudoinformation verpflichtet sei. Mehrere Entwicklungen haben die Medienlandschaft radikal verändert. Roger de Weck nennt die elektronische Revolution, den Umbruch zur Dienstleistungsgesellschaft, die Globalisierung und das Ende der bipolaren Welt. Weil es für die heutigen Medien weit mehr Vertriebskanäle als Nachrichtenstoff gebe, herrsche ein Verteilungskampf, der mit den Mitteln der Boulevardisierung, der Wiederholung des Immergleichen, Erschliessung neuer Themen und Erfindung von Kunstwelten, wie Events, Reality-TV oder Computerspielen, ausgetragen werde. Der frühere Chefredaktor ortet im Abbau von Auslandskorrespondenten den Rückzug ins Provinzielle, trendige Lifestyleinhalte ersetzen Sozialreportagen und Hintergrundberichte. Dem «content provider» fehlen Offenheit und Neugierde für tieferliegende politische Ursachen. Der kurzlebigen Erregungskultur entspricht die Interessenverbindung mit parteilichem Populismus. Das charakteristische Element der Massenmedien ist die Show. Ihr ist die Verpackung wichtiger als der Inhalt. Auf das noch $\mathrm{zu}$ entdeckende Neue wird wie auf alles Nichtdarstellbare verzichtet, weil es zu kompliziert, noch ungestaltet und formlos und damit der Aufwand zu gross ist. Guter Journalismus sollte kritisch und nicht affirmativ, kompetent und nicht irrational, selbstkritisch und nicht auf sich bezogen sein.

Peter Stulz, Chefarzt für Herz-, Thorax- und Gefässchirurgie, demonstrierte das Gesagte mit einer Filmwiedergabe aus der «Puls»-Sendung «Herznacht». Szenen aus dem Operationssaal wechseln mit unbeholfenen Gesprächsversuchen am runden Tisch mit Angehörigen. Die 
Fernsehdramaturgie begnügt sich mit dürftigen Kommentaren, sie arbeitet assoziativ und emotional. Zelebriert wird das aseptische Ritual einer High-Tech-Inszenierung, die Schnittwechsel suggerieren die Hektik einer Sportreportage. Das voyeurhafte Spiel gibt vor, die Öffentlichkeit aufzuklären, doch erklärt wird nichts, der medizinische Informationsgehalt ist gleich null. Kamera und Drehbuch folgen exakt der von Medienprofis zuvor erklärten Dynamik. Die Privatsphäre wird dabei systematisch verletzt, der Patient zum Objekt reduziert, die persönlichen Wünsche und Ängste, die Intimität des Gesprächs, existentielle Vertrauensfragen, die Probleme der informierten Zustimmung, das alles bleibt ausgeblendet. Das Team im Operationssaal und das Team im Studio kommen nicht wirklich miteinander ins Gespräch. Dem Geltungsbedürfnis narzisstischer Ärzte kommt der Profilierungszwang der Spitäler entgegen. Das Geschäft verlangt Werbung, denn wer im verordneten Wettbewerb bestehen will, muss sich bei jeder Gelegenheit in Erinnerung rufen. Für den Referenten ist klar, dass Pflegende und Ärzte die Patienten vor dieser Betriebs- und Medienlogik zu schützen haben, auch wenn sie damit ziemlich quer in der Landschaft liegen.

Erweitert wurden diese Kernthemen durch Beiträge zum Wissenschaftsjournalismus, zur Tätigkeit des Schweizer Presserates (empfehlenswert: www.presserat.ch), zur medialen Inszenierung von Medizin am Beispiel des Klonens, zum Arztbild im Film, zum Patienten im Informationsdschungel, zu philosophischen Aspekten der Wahrnehmung und zu Sprachlosigkeit von Schmerz und Leiden. The message, frei nach McLuhan: Etwas Medienkunde kann Ärztinnen und Ärzten nicht schaden. 